\title{
Chemicals to enhance microalgal growth and accumulation of high-value bioproducts
}

\author{
Xinheng Yu ${ }^{1,2,3}$, Lei Chen ${ }^{1,2,3}$ and Weiwen Zhang ${ }^{1,2,3}$ * \\ ${ }^{1}$ Laboratory of Synthetic Microbiology, School of Chemical Engineering and Technology, Tianjin University, Tianjin, China \\ ${ }^{2}$ Key Laboratory of Systems Bioengineering (Ministry of Education), Tianjin University, Tianjin, China \\ ${ }^{3}$ SynBio Research Platform, Collaborative Innovation Center of Chemical Science and Engineering (Tianjin), Tianjin, China
}

\section{Edited by:}

Maria De Angelis, University of Bari

Aldo Moro, Italy

\section{Reviewed by:}

Ebrahim Hadavi, Islamic Azad

University, Iran

Zhao Chen, Clemson University, USA

\section{*Correspondence:}

Weiwen Zhang, Laboratory of Synthetic Microbiology, School of Chemical Engineering and Technology, Tianjin University, Tianjin 300072, China

e-mail:wwzhang8@tju.edu.cn
Photosynthetic microalgae have attracted significant attention as they can serve as important sources for cosmetic, food and pharmaceutical products, industrial materials and even biofuel biodiesels. However, current productivity of microalga-based processes is still very low, which has restricted their scale-up application. In addition to various efforts in strain improvement and cultivation optimization, it was proposed that the productivity of microalga-based processes can also be increased using various chemicals to trigger or enhance cell growth and accumulation of bioproducts. Herein, we summarized recent progresses in applying chemical triggers or enhancers to improve cell growth and accumulation of bioproducts in algal cultures. Based on their enhancing mechanisms, these chemicals can be classified into four categories:chemicals regulating biosynthetic pathways, chemicals inducing oxidative stress responses, phytohormones and analogs regulating multiple aspects of microalgal metabolism, and chemicals directly as metabolic precursors. Taken together, the early researches demonstrated that the use of chemical stimulants could be a very effective and economical way to improve cell growth and accumulation of high-value bioproducts in large-scale cultivation of microalgae.

Keywords: chemicals, microalgae, growth, accumulation, bioproducts

\section{INTRODUCTION}

Microalgae are autotrophic organisms, which utilize light energy, and inorganic nutrients such as $\mathrm{CO}_{2}$, nitrogen and phosphorus, to generate biomass and synthesize valuable metabolites. Some algal species cultivated under stress conditions accumulate specific secondary metabolites (i.e., pigments, vitamins, or lipids), which are high-value bioproducts that can be applied in the cosmetic, food, or pharmaceutical sectors (Skjanes et al., 2013). In contrast to higher plants that contain large amount of cellulose and hemicellulose, larger portion of algal biomass can be directly converted into biofuels or other high-value bioproducts via downstream processes (Wijffels et al., 2010; Vanthoor-Koopmans et al., 2013; Yen et al., 2013). One well-known area of such applications is microalgae-based biodiesel that has been proposed as good alternative to non-renewable fossil fuels (Sheehan et al., 1998), and another area of commercial exploitation of microalgae is the production of pharmaceutically and high-value industrial chemicals (Leu and Boussiba, 2014).

Although microalgae are known to synthesize a variety of bioproducts with potential commercial values, only a few of them, such as $\beta$-carotene and astaxanthin, have been produced at an industry-scale (Ben-Amotz, 1995; Sheehan et al., 1998; Borowitzka, 2013), which may be due to the low productivity of these products in the native microalgae and the difficulty in isolating them by economically feasible means (Clarens et al., 2010; Norsker etal., 2011; Razon and Tan, 2011; Soratana and Landis, 2011). While significant efforts have been undertaken to select high-yield strains, optimize cultivation and even modify the strains by genetic engineering in the past decades (Suen et al., 1987; Cerón Garcìa et al., 2005; Kilian et al., 2011), progress has yet to be fully satisfied.

As an alternative method to improve production or accumulation of bioproducts, chemicals as metabolic triggers or enhancers that are able to directly modulate cellular metabolism have been proposed and applied in various commercially viable microalgae. Unlike genetic modification, this approach relies on phenotypic screening and does not require specific knowledge of molecular targets in metabolic and catabolic pathways involved in synthesis of bioproducts. In a recent study, Franz etal. (2013) described a phenotypic screening of 42 chemicals for their roles on lipid metabolism in microalgae, and identified 12 chemicals that are capable of enhancing intracellular lipid levels by $>100 \%$, with three compounds (i.e., epigallocatechin gallate, CDK2 inhibitor 2 and cycloheximide) increasing intracellular lipids by $200-400 \%$ based on Nile Red fluorescence intensity. In addition, the researchers took a further step to verify these chemicals in large-scale cultures and concluded that propyl gallate and butylated hydroxyanisole could be used in large-scale applications considering the low cost of the chemicals and the lipid content increases (Franz etal., 2013), demonstrating that the application of chemical enhancer could be a valuable and practical approach in addressing the low productivity issue with microalgae-based processes. In this article, we review the recent progresses in applying chemicals to improve cell growth and accumulation of high-value bioproducts in microalgae (Table 1), with a focus on the molecular mechanisms of their stimulatory roles. 


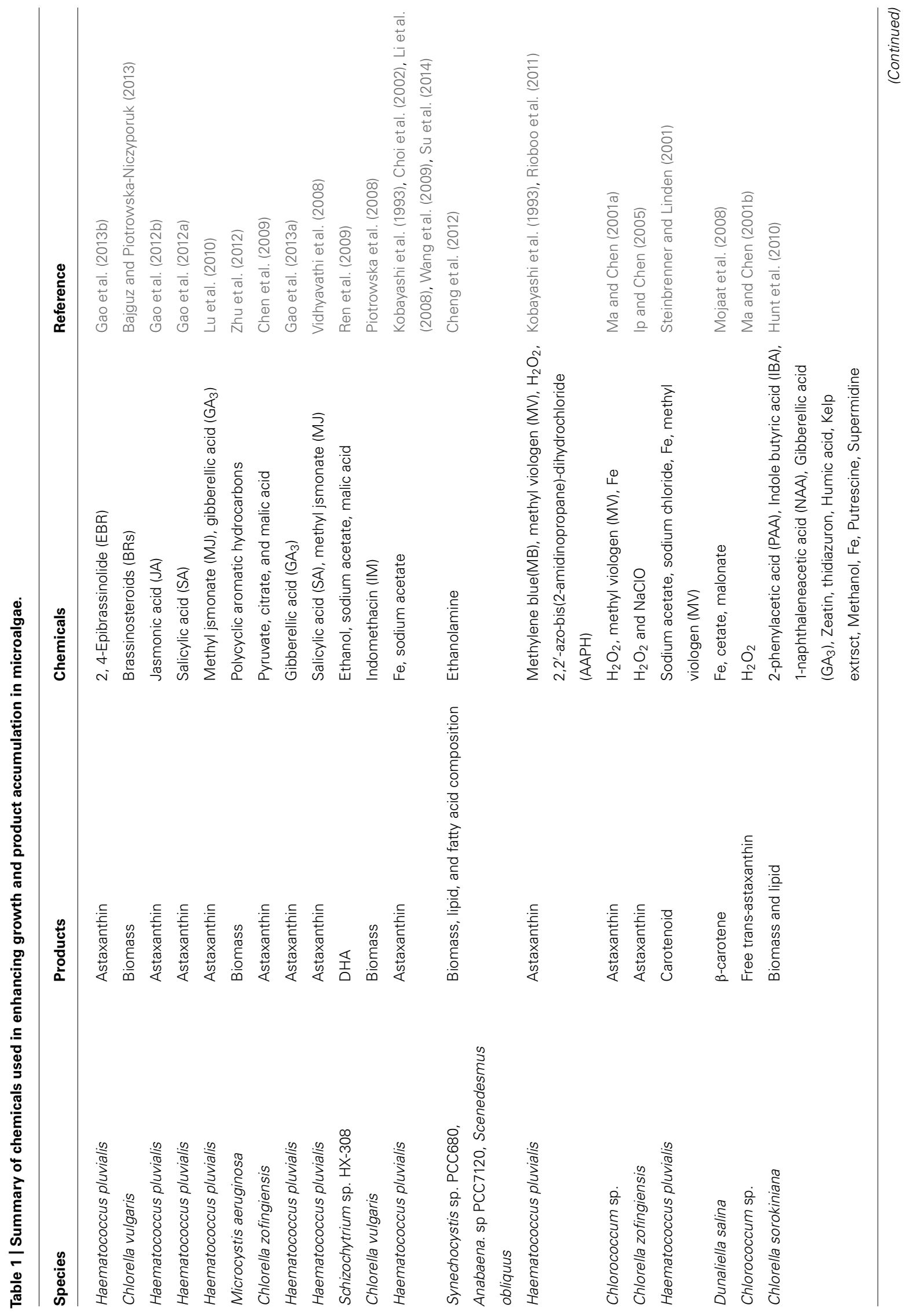




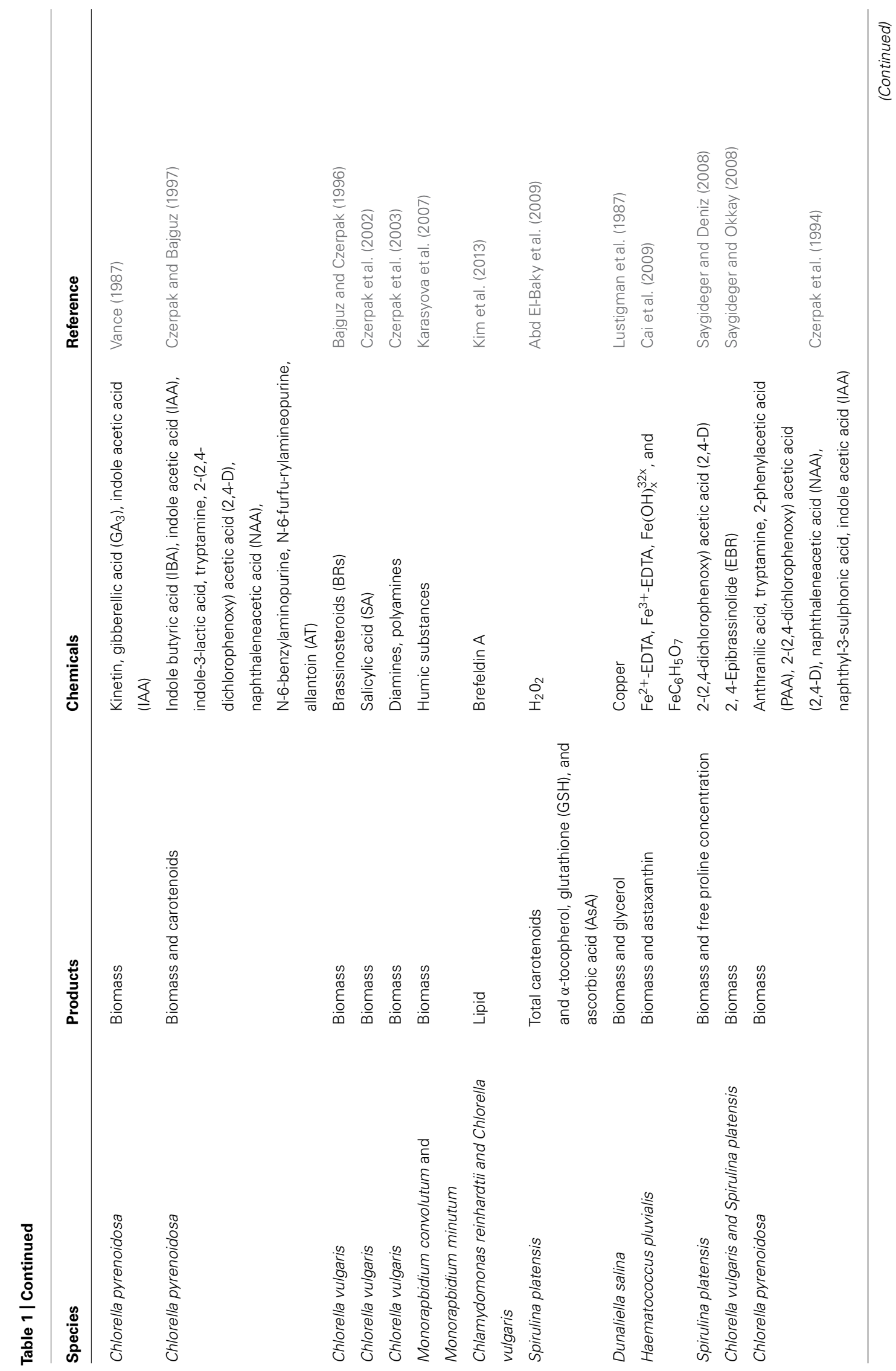




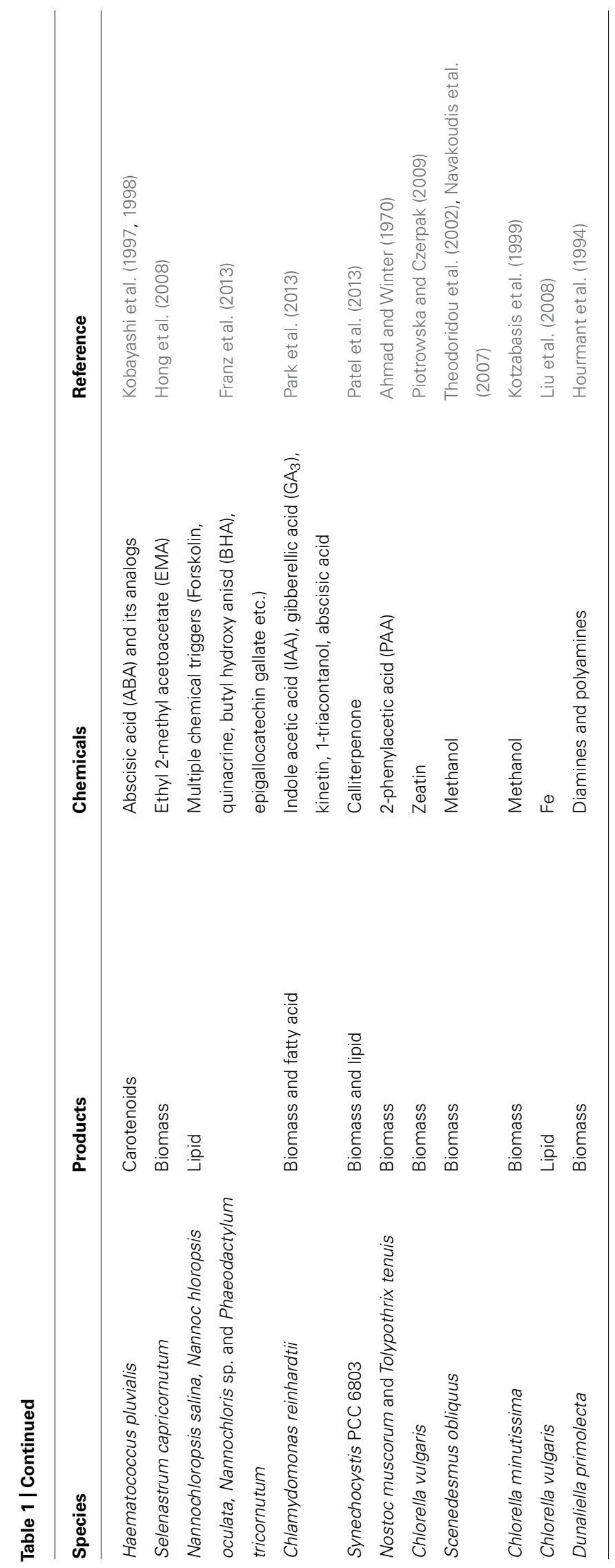




\section{PHYTOHORMONES AND ANALOGS REGULATING MULTIPLE ASPECTS OF METABOLISM \\ TARGETING ON BIOSYNTHETIC PATHWAYS OF HIGH-VALUE PRODUCTS}

It has been established that plants have developed a broad spectrum of molecular mechanisms to resist unfavorable environmental perturbations (Ren et al., 2009). Microalgae that share the evolutionary merits with plants also have mechanisms to deal with various environmental stress. One well-studied example is antioxidant pigment astaxanthin that plays a critical role in response to various stress conditions, such as high light, salinity, nutrient stress, and high carbon/nitrogen ratio, in chlorophyceae Haematococcus pluvialis (Tripathi et al., 1999; Sarada et al., 2002). The pathway of astaxanthin synthesis in $H$. pluvialis has been deciphered (Grünewald et al., 2000; Vidhyavathi et al., 2008) and several biosynthetic genes related to carotenoid have also been cloned and characterized (Lotan and Hirschberg, 1995; Sun et al., 1998; Linden, 1999; Steinbrenner and Linden, 2003; Huang et al., 2006). To increase the astaxanthin productivity, chemicals as metabolism enhancers were also evaluated recently. In one study, Lu et al. (2010) reported that gibberellic acid $\left(\mathrm{GA}_{3}\right)$ and methyl jsmonate $(\mathrm{MJ})$ played roles in regulating gene expression of bkts that catalyzes $\beta$-carotene to canthaxanthin in the astaxanthin biosynthetic pathway (Lu et al., 2010). More recently, Gao et al. (2012a,b, 2013a,b) found that chemicals jasmonic acid (JA), salicylic acid (SA), GA 3 , and 2, 4-epibrassinolide (EBR) can enhance astaxanthin production to $1.458 \mathrm{mg} / \mathrm{L}, 2.74 \mathrm{mg} / \mathrm{L}$, $2.39 \mathrm{mg} / \mathrm{L}, 2.26 \mathrm{mg} / \mathrm{L}$, respectively; and further analysis showed that the enhancing mechanisms of chemicals were concentrationdependent. For example, the results showed that $25 \mathrm{mg} / \mathrm{L} \mathrm{JA}$ up-regulated the transcriptional expression of $p d s, c r t \mathrm{R}-\mathrm{B}$, and $l y c$ of the astaxanthin biosynthetic pathway ( $>10$-fold up-regulation) the most, while $50 \mathrm{mg} / \mathrm{L} \mathrm{JA}$ impacted the transcriptional expression of ipi-1, ipi-2, psy, crt R-B, and $c r t \mathrm{O}$ than on $p d s$, lyc, and bkt2 more significantly (Gao et al., 2012b). Based on a correlation analysis between their maximum mRNA transcripts of five carotenoid genes and astaxanthin production, Li et al. (2010) proposed that multiple regulatory mechanisms at transcriptional, translational, and post-translational levels of astaxanthin biosynthetic genes co-existed in controlling the overall carotenogenesis process in $\mathrm{H}$. pluvialis (Li et al., 2010). Interestingly, different modes of regulation can be issued by the same chemical in $H$. pluvialis, such as JA that up-regulated $p s y, p d s, c r t \mathrm{R}-\mathrm{B}, l y c, b k t$, and $c r t \mathrm{O}$ genes at the transcriptional level, and up-regulated $i p i-1$ and $i p i-2$ genes at both transcriptional and post-transcriptional levels, respectively; and SA up-regulated ipi-1, ipi-2, psy, crtR$\mathrm{B}, b k t$, and $c r t \mathrm{O}$ gene at the transcriptional level, and $l y c$ at the post-transcriptional level and $p d s$ at both levels, respectively (Gao et al., 2012a,b).

\section{INDUCING OXIDATIVE STRESS RESPONSES}

Photosynthetic algae, like higher plants, generate reactive oxygen species (ROS) through chloroplast photosynthesis and mitochondrial respiration under stress condition, and ROS will then to be used as signal molecules to initiate production and accumulation of many bioproducts (Asada, 1994). The effects of SA and MJ on the antioxidant systems in $H$. pluvialis were investigated, and the results showed that at low concentrations, $100 \mu \mathrm{M}$ SA increased astaxanthin content to 6.8 -fold under low light $(30 \mu \mathrm{mol}$ $\mathrm{m}^{-2} \mathrm{~s}^{-1}$ ), while $10 \mu \mathrm{M} \mathrm{MJ}$ showed marginal increase in astaxanthin. However, at high concentration of $500 \mu \mathrm{M}$, both SA and $\mathrm{MJ}$ reduced the growth of microalgae and inhibited astaxanthin accumulation. Further mechanism analysis showed that SA at high concentrations increased superoxide dismutase activity to 4.5- and 3.3-fold and ascorbate peroxidase (APX) activity to 15.5- and 7.1-fold under low and high light, respectively, while MJ increased catalase activity (1.4-fold) under high light and APX activity (5.4-fold) under low light, suggesting the low astaxanthin accumulation may be due to the free radicals being scavenged (Raman and Ravi, 2010).

\section{REGULATING OTHER ASPECTS OF CELLULAR METABOLISM}

Phytohormones are signal molecules synthetized by plants, and capable of efficiently regulating cellular metabolism at very low concentrations (Park et al., 2013). The application of phytohormones to improve growth and productivity has been reported, and the results with Chlorella species showed that use of natural and synthetic auxins, as well as their precursors, have considerable stimulating effects on algal growth and biomass composition (Czerpak et al., 1994, 1999; Czerpak and Bajguz, 1997; Hunt et al., 2010). In addition, a combination of chemicals from within the auxin family as well as with that of other families, such as $5 \mathrm{ppm}$ 1-naphthaleneacetic acid (NAA) $+10 \mathrm{ppm} \mathrm{GA}_{3}+1 \mathrm{ppm}$ zeatin (ZT), dramatically increased biomass productivity by $170 \%$ over the control in Chlorella sorokiniana (Hunt et al., 2010). Another study investigated the effects of phytohormones on microalgal growth and oil accumulation for biodiesel production in Chlamydomonas reinhardtii. The results indicated that all five of the tested phytohormones (i.e., indole-3-acetic acid, gibberellic acid, kinetin, 1-triacontanol, and abscisic acid) promoted cell growth. In particular, hormone treatment increased biomass production by $54-69 \%$ relative to the control growth medium, demonstrating their values in decreasing cost of commercial biodiesel production (Park et al., 2013).

Brassinosteroids (BRs) are hydroxylated derivatives of 5cholestane and important plant growth regulators in multiple developmental processes, such as cell division and cell elongation (Bajguz and Czerpak, 1996; Bajguz and Tretyn, 2003). A recent study found that BRs cooperated synergistically with auxins in stimulating cell proliferation and endogenous accumulation of proteins, chlorophylls, and monosaccharides in C. vulgaris (Bajguz and Piotrowska-Niczyporuk, 2013).

In terms of the molecular mechanisms, auxins and their analogs have been found to affect photosynthetic efficiency and $\mathrm{CO}_{2}$ fixation in microalgae. For example, a study showed that auxins had incentive effects on reactions of bonding $\mathrm{CO}_{2}$ to 1, 5-biphosphoribulose and photosynthetic phosphorylation. As expected, the increase in intensity of photosynthesis reactions correlated well with higher contents of chlorophylls, pheophytins, and total carotenoids in cells treated with indomethacin that shares structural similarity with natural auxins (Piotrowska et al., 2008). Other studies also indicated that low concentrations of synthetic auxins, such as 2-(2,4-dichlorophenoxy) acetic acid (2,4-D), NAA and 2-phenylacetic acid (PAA), stimulated the photosynthetic rate and chlorophylls as well as carotenoids synthesis in green 
algae C. pyrenoidosa, Scenedesmus acuminatus, and S. qadricauda (Czerpak et al., 1994, 1999, 2002; Wong, 2000).

Diamines and polyamines are polycation nitrogen compounds presented in almost all prokaryotic and eukaryotic microorganisms and belonged to specific cellular regulators of growth and metabolism (Rayle and Cleland, 1992). The study showed that in C. vulgaris treated with diamines and polyamines, the content of monosaccharides, primary products of Calvin cycle were intensively stimulated on 3 days of $C$. vulgaris culture, while chlorophyll content was enhanced on 9 days of $C$. vulgaris culture, indicating that the amines stimulated the dark phase of photosynthesis in the young cells, and the light synthesis phase in aging cells, respectively (Czerpak et al., 2003).

An acid growth theory has been proposed to explain the cell elongation triggered by auxins in plant cells, which refers to the auxin-induced acidification of free space in cell wall. The decrease of $\mathrm{pH}$ enhances the plasticity of cell wall thus contributes to the increased elongation rate of the plant tissues, and the phenomenon is presumably related to the activation of membrane-binding proton pumps by auxin (Rayle and Cleland, 1992; Hobbie et al., 1994). A study with algal C. vulgaris also showed that BR-stimulated cell growth depended at least partly on acid growth theory (Bajguz and Czerpak, 1996).

Cell phase and mitosis regulated by phytohormones was also reported in microalgae. A recent study showed that NAA (30 ppm) treatment stimulated higher biomass productivity between days 5 and 10 while PAA ( 5 ppm) treatment effected on the first 5 days in in C. sorokiniana, suggesting that NAA might prolong exponential phase and PAA might short initial lag phase before initiation of cell division. The combination of NAA (5 ppm) + PAA (30 ppm) showed 104\% increase of biomass and demonstrated that auxins enhanced biomass growth by reducing generation time thus contributing to reducing generation time (Hunt et al., 2010). Another study on the synchronous culture of C. pyrenoidosa showed that the time to incipient cell division was reduced by GA and 6furfurylaminopurine, suggesting these two phytohormones had played roles in eliminating the initial lag phase (Vance, 1987). Similarly, the cell number and dry weight of C. vulgaris was also significantly increased in response to optimal dose of IM $\left(10^{-7}\right.$ $\mathrm{M})$ on a 5-day cultivation, suggesting that growth elicited by natural and synthetic auxins encompassed the stimulation of mitosis (Piotrowska et al., 2008).

Chlorophyll pigment presents challenges to lipid extraction and biodiesel conversion in downstream processing of algal biomass. Hence, chemicals led to higher biomass and lower pigment production will bring benefits. A study showed that the addition of NAA (30 ppm) and PAA (5 ppm) significantly increased biomass production, meanwhile decreased chlorophyll a synthesis in C. sorokiniana (Hunt et al., 2010). In addition, auxins at high concentrations can activate key regulatory enzyme in ethylene biosynthesis (Grossmann, 2000), and large amount of ethylene could then induce the degradation of photosynthetic pigments (Sunohara and Matsumoto, 1997).

As for other regulatory functions, an exposure of C. vulgaris cells to exogenous IM, synthetic analog of IAA, has been reported to increase cellular DNA level up to $48 \%$ and $20-43 \%$ more soluble proteins excreted to the environments (Piotrowska et al.,
2008); and cytokinins and allantoin (AT) were found to stimulate carotenoids content by $185-190 \%$ and $124 \%$ in C. pyrenoidosa, possibly due to their inhibition of oxidases and dehydrogenases that are responsible for oxidation process and degradation of chlorophylls and carotenoids (Czerpak and Bajguz, 1997).

\section{OTHER CHEMICALS INDUCING OXIDATIVE STRESS RESPONSES}

Apart from phytohormones and analogs, other chemicals capable of inducing oxidative response for enhanceing microalgal growth and accumulation of high-value bioproducts were also investigated. An early study showed that $\mathrm{Fe}^{2+}$, methylene blue (MB) for singlet oxygen $\left({ }^{1} \mathrm{O}_{2}\right)$, methyl viologen (MV) for superoxide anion radical $\left(\mathrm{O}_{2}{ }^{-}\right), \mathrm{H}_{2} \mathrm{O}_{2}$, and 2,2'-azo-bis(2-amidinopropane)dihydrochloride $(\mathrm{AAPH})$ for peroxy radical $\left(\mathrm{AO}_{2} \cdot\right)$, were capable of triggering astaxanthin biosynthesis in $H$. pluvialis, in which $\mathrm{Fe}^{2+}$ possibly served as an $\mathrm{HO}$. generator via an ironcatalyzed Fenton reaction (Kobayashi et al., 1993). HO. or other active oxygen species $\left({ }^{1} \mathrm{O}_{2}, \mathrm{O}_{2}{ }^{-}, \mathrm{H}_{2} \mathrm{O}_{2}\right.$, and $\left.\mathrm{AO}_{2} \cdot\right)$ might then enhance carotenoid formation in algal cyst cells by participating directly in the carotenogenic enzyme reactions as an oxidizer or an $\mathrm{H}$ acceptor (Beyer and Kleinig, 1989). In a recent study, Ip and Chen (2005) proposed sodium hypochlorite $(\mathrm{NaClO})$ as another oxygen species to enhance astaxanthin production of $C$. zofingiensis in the heterotrophic cultivation medium.

\section{CHEMICALS AS METABOLIC PRECURSORS}

An early study showed that an addition of $100 \mathrm{mM}$ pyruvate into the culture medium of $C$. zofingiensis enhanced the yield of astaxanthin from 8.36 to $10.72 \mathrm{mg} / \mathrm{L}$. In addition, citrate and malic acid also had the similar stimulatory effects on the formation of astaxanthin. Pyruvate might serve as a precursor for isopentenyl pyrophosphate (IPP), the carotenoid precursor in C. zofingiensis and $H$. pluvialis, while the stimulatory effects of citrate and malic acid on astaxanthin biosynthesis in C. zofingiensis could be due to their conversions to pyruvate (Chen et al., 2009). For docosahexaenoic acid (DHA) accumulation in Schizochytrium sp. HX-308, an addition of $4 \mathrm{~g} / \mathrm{L}$ malic acid to the culture medium at the rapid lipid accumulation stage can increase DHA content of total fatty acids from 35 to $60 \%$. In addition to functioning as a possible carbon precursor, it was speculated that malic acid added at rapid lipid accumulation stage could activate malic enzyme activity and enhance NADPH generating reaction from malic acid to pyruvate (Ren et al., 2009). In addition, ethanol was also found to enhance lipid content by 35\% in Crypthecodinium cohnii, in which ethanol can be converted to acetyl-CoA directly and in its metabolism might generate additional reducing power NADPH for lipogenesis (Lolke et al., 2005).

To aid in identifying metabolites associated with enhanced production of bioproducts, metabolomics, a measurement, and study of the small-molecule metabolites that constitute cellular metabolic networks, has been recently applied. In one study, Cheng etal. (2012) compared the metabolites between two cyanobacteria Synechocystis sp. PCC6803 and Anabaena sp. PCC 7120, and one microalga S. obliquus by gas chromatography coupled with time-of-flight mass spectrometry to 
detect important metabolites intricately tied to the lipid content in cyanobacteria and microalgae. The results showed that nine metabolites including ethanolamine were associated with the different lipid accumulation, and further study confirmed that addition of exogenous ethanolamine $(2 \mathrm{mmol} / \mathrm{L})$ could increase the lipid content by $22 \%$ in S. obliquus (Cheng et al., 2012). In another study, Su et al. (2014) investigated mechanism of astaxanthin induction under various stress conditions using a metabolomics and network analysis, and found that several metabolites, such as $\mathrm{D}$ - $(+)$ altrose, D-ribose 5-phosphate, $L$-glutamic acid, and $\alpha$-ketoglutaric acid, were positively associated with the increased astaxanthin accumulation in $H$. pluvialis. Although further confirmation is still needed, it was speculated that the increased abundances of these metabolites might contribute to the enhanced carbon flow into the astaxanthin biosynthesis (Su et al., 2014). Taken together, these early studies demonstrated that metabolomics could be a valuable tool in identifying potential metabolites for enhancing target production in algae (Zhang et al., 2010). Effective mechanisms of the chemicals were schemed in Figure 1.

\section{CONCLUSION}

To produce bioproducts form microalgae in an economically feasible and sustainable way, one major hurdle that needs to be overcome is the low productivity. To address the issues, efforts have been undertaken to identify and apply chemical triggers or enhancers to enhance cell growth and accumulation of bioproducts in microalgae, and the studies have demonstrated that application of chemical triggers or enhancers could be a very practical method in large-scale fermentation of microalgae. In addition, the possible stimulatory mechanisms were also partially deciphered for some of the chemicals. However, to uncover new chemicals and expand the application, it is necessary to determine more accurately the metabolic mechanisms related to cell growth,

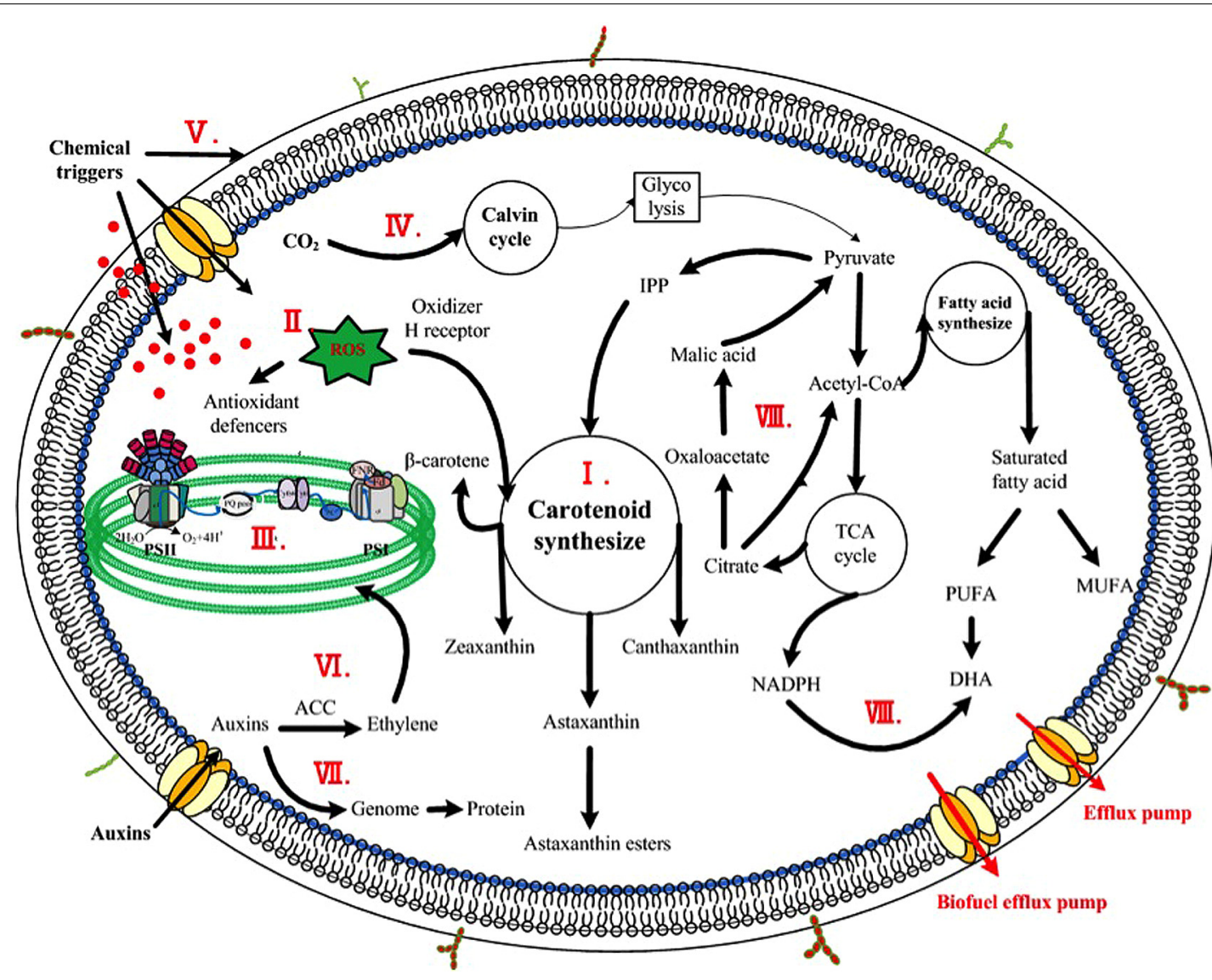

FIGURE 1 | Scheme of enhancing mechanisms of chemicals on microalgae. The major stimulatory mechanisms were indicted inside the cell. (I) Chemicals targeting on biosynthetic pathways of high-value product, such as JA, SA, GA, and EBR controlling the overall carotenogenesis process in $H$. pluvialis; (II) Chemicals inducing oxidative stress responses, including direct or indirect addition of active oxygen species and chemical triggers inducing antioxidant production; (III) Phytohormones and analogs effecting on photosynthetic efficiency, namely the light phase, including photosynthetic phosphorylation, photosynthetic rate, and chlorophylls synthesis; (IV) Phytohormones and analogs impacting $\mathrm{CO}_{2}$ fixation, namely the dark phase of photosynthesis, such as diamines and polyamines stimulating production of Calvin cycle; (V) Phytohormones and analogs encompassed acid growth theory, alternating the plasticity of cell wall thus contributing to cell elongation; (VI) Degradation of photosynthetic pigments due to large amount of ethylene caused by high concentration of auxins; (VII) Phytohormones and analogs regulating genome and protein expression, such as IM modulating DNA and protein content in C. vulgaris; (VIII) Chemicals as metabolic precursors, such as pyruvate serving as a precursor of carotenoid synthesis thus stimulating the formation of astaxanthin and NADPH (led by malic acid) acting as a precursor of fatty acid synthesis increasing DHA content. 
production and accumulation of bioproducts, and the modes of action (MOA) of chemicals in microalgae. For this regard, the application of various global-focused technologies, such as genomics, proteomics, and metabolomics, could be valuable tools in the future research.

\section{ACKNOWLEDGMENTS}

The research was supported by grants from the National High-tech R\&D Program (No. 2012AA02A707), and the Doctoral Program of Higher Education of China (No. 20120032110020 and 20130032120022).

\section{REFERENCES}

Abd El-Baky, H., El Baz, F., and El-Baroty, G. (2009). Enhancement of antioxidant production in Spirulina platensis under oxidative stress. Acta physiol. plant. 31, 623-631. doi: 10.1007/s11738-009-0273-278

Ahmad, M., and Winter, A. (1970). The effect of weak auxins on the growth of blue-green algae. Hydrobiologia 36, 305-316. doi: 10.1007/bf00035329

Asada, K. (1994). "Production and action of active oxygen species in photosynthetic tissues," in Causes of Photooxidative Stress and Amelioration of Defense Systems in Plants, eds C. H. Foyer and P. M. Mullineaux (Boca Raton, FL: CRC Press), $77-104$.

Bajguz, A., and Czerpak, R. (1996). Effect of brassinosteroids on growth and proton extrusion in the alga Chlorella vulgaris Beijerinck (Chlorophyceae). J. Plant Growth Regul. 15, 153-156. doi: 10.1007/bf00198931

Bajguz, A., and Piotrowska-Niczyporuk, A. (2013). Synergistic effect of auxins and brassinosteroids on the growth and regulation of metabolite content in the green alga Chlorella vulgaris (Trebouxiophyceae). Plant Physiol. Biochem. 71, 290-297. doi: 10.1016/j.plaphy.2013.08.003

Bajguz, A., and Tretyn, A. (2003). The chemical characteristic and distribution of brassinosteroids in plants. Phytochemistry 62, 1027-1046. doi: 10.1016/S00319422(02)00656-8

Ben-Amotz, A. (1995). New mode of Dunaliella biotechnology: two-phase growth for $\beta$-carotene production. J. Appl. Phycol. 7, 65-68. doi: 10.1007/bf00003552

Beyer, P., and Kleinig, H. (1989). "On the desaturation and cyclization reactions of carotenes," in Chromoplast Membranes, Carotenoids, eds N. Krinsky, M. MathewsRoth, and R. Taylor (New York, NY: Springer), 195-206.

Borowitzka, M. (2013). High-value products from microalgae-their development and commercialisation. J. Appl. Phycol. 25, 743-756. doi: 10.1007/s10811-0139983-9989

Cai, M., Li, Z., and Qi, A. (2009). Effects of iron electrovalence and species on growth and astaxanthin production of Haematococcus pluvialis. Chin. J. Oceanol. Limnol. 27, 370-375. doi: 10.1007/s00343-009-9176-9171

Cerón Garcìa, M., Sánchez Mirón, A., Fernández Sevilla, J., Molina Grima, E., and Garci ìa Camacho, F. (2005). Mixotrophic growth of the microalga Phaeodactylum tricornutum. Process Biochem. 40, 297-305. doi: 10.1016/j.procbio.2004.01.016

Chen, T., Wei, D., Chen, G., Wang, Y., and Chen, F. (2009). Employment of organic acids to enhance astaxanthin formation in heterotrophic Chlorella zofingensis. J. Food Process. Preserv. 33, 271-284. doi: 10.1111/j.1745-4549.2009.00391.x

Cheng, J., Niu, Y., Lu, S., and Yuan, Y. (2012). Metabolome analysis reveals ethanolamine as potential marker for improving lipid accumulation of model photosynthetic organisms. J. Chem. Technol. Biotechnol. 87, 1409-1418. doi: 10.1002/jctb.3759

Choi, Y., Yun, Y., and Park, J. (2002). Evaluation of factors promoting astaxanthin production by a unicellular green alga, Haematococcus pluvialis, with fractional factorial design. Biotechnol. Prog. 18, 1170-1175. doi: 10.1021/bp025549b

Clarens, A., Resurreccion, E., White, M., and Colosi, L. (2010). Environmental life cycle comparison of algae to other bioenergy feedstocks. Environ. Sci. Technol. 44 1813-1819. doi: 10.1021/es902838n

Czerpak, R., and Bajguz, A. (1997). Stimulatory effect of auxins and cytokinins on carotenes, with differential effects on xanthophylls in the green alga Chlorella pyrenoidosa Chick. Acta Soc. Bot. Pol. 66, 41-46. doi: 10.5586/asbp.1997.006

Czerpak, R., Bajguz, A., Białecka, B., Wierzchołowska, L., and Wolañska, M. (1994). Effect of auxin precursors and chemical analogues on the growth and chemical composition in Chlorella pyrenoidosa Chick. Acta Soc. Bot. Pol. 63, 279-286. doi: 10.5586/asbp. 1994.038
Czerpak, R., Dobrzyn, P., Krotke, A., and Kicinska, E. (2002). The effect of auxins and salicylic acid on chlorophyll and carotenoid contents in Wolffia arrhiza (L.) Wimm. (Lemnaceae) growing on media of various trophicities. Pol. J. Environ. Stud. 11, 231-235.

Czerpak, R., Krotke, A., and Mical, A. (1999). Comparison of stimulatory effect of auxins and cytokinins on protein, saccharides and chlorophylls content in Chlorella pyrenoidosa Chick. Pol. Arch. Hydrobiol. 46, 71-82.

Czerpak, R., Piotrowska, A., Dobrogowska, R., Matejczyk, M., and Wieslawski, W. (2003). Biochemical activity of di-and polyamines in the green alga Chlorella vulgaris Beijerinck (Chlorophyceae). Acta soc. bot. Pol. 72, 19-24. doi: 10.5586/asbp.2003.003

Franz, A., Danielewicz, M., Wong, D., Anderson, L., and Boothe, J. (2013). Phenotypic screening with oleaginous microalgae reveals modulators of lipid productivity. ACS Chem. Biol. 8, 1053-1062. doi: 10.1021/ cb300573r

Gao, Z., Meng, C., Gao, H., Li, Y., Zhang, X., Xu, D., et al. (2013a). Carotenoid genes transcriptional regulation for astaxanthin accumulation in fresh water unicellular alga Haematococcus pluvialis by gibberellin A3 (GA3). Indian J. Biochem. Biophys. $50,548-553$.

Gao, Z., Meng, C., Gao, H., Zhang, X., Xu, D., Su, Y., et al. (2013b). Analysis of mRNA expression profiles of carotenogenesis and astaxanthin production of Haematococcus pluvialis under exogenous 2, 4-epibrassinolide (EBR). Biol. Res. 46, 201-206. doi: 10.4067/S0716-97602013000200012

Gao, Z., Meng, C., Zhang, X., Xu, D., Miao, X., Wang, Y., et al. (2012a). Induction of salicylic acid (SA) on transcriptional expression of eight carotenoid genes and astaxanthin accumulation in Haematococcus pluvialis. Enzyme Microb. Technol. 51, 225-230. doi: 10.1016/j.enzmictec.2012.07.001

Gao, Z., Meng, C., Zhang, X., Xu, D., Zhao, Y., Wang, Y., et al. (2012b). Differential expression of carotenogenic genes, associated changes on astaxanthin production and photosynthesis features induced by JA in H. pluvialis. PLoS ONE 7:e42243. doi: 10.1371/journal.pone.0042243

Grossmann, K. (2000). Mode of action of auxin herbicides: a new ending to a long, drawn out story. Trends Plant Sci. 5, 506-508. doi: 10.1016/S13601385(00)01791-x

Grünewald, K., Eckert, M., Hirschberg, J., and Hagen, C. (2000). Phytoene desaturase is localized exclusively in the chloroplast and up-regulated at the mRNA level during accumulation of secondary carotenoids in Haematococcus pluvialis (Volvocales, Chlorophyceae). Plant Physiol. 122, 1261-1268. doi: 10.1104/pp.122.4.1261

Hobbie, L., Timpte, C., and Estelle, M. (1994). "Molecular genetics of auxin and cytokinin," in Signals and Signal Transduction Pathways in Plants, ed. K. Palme (Amsterdam: Springer), 263-283. doi: 10.1007/978-94-011-0239-1_15

Hong, Y., Hu, H., and Li, F. (2008). Growth and physiological responses of freshwater green alga Selenastrum capricornutum to allelochemical ethyl 2-methyl acetoacetate (EMA) under different initial algal densities. Pestic. Biochem. Physiol. 90, 203-121. doi: 10.1016/j.pestbp.2007.11.009

Hourmant, A., Mereau, N., Penot, M., Cann, C., and Caroff, J. (1994). Influence of polyamines on growth and metabolism of Dunaliella primolecta. Acta Bot. Neerlandica. 43, 129-136. doi: 10.1111/j.1438-8677.1994. tb00740.x

Huang, J., Chen, F., and Sandmann, G. (2006). Stress-related differential expression of multiple $\beta$-carotene ketolase genes in the unicellular green alga Haematococcus pluvialis. J. Biotechnol. 122, 176-185. doi: 10.1016/j.jbiotec.2005. 09.002

Hunt, R., Chinnasamy, S., Bhatnagar, A., and Das, K. (2010). Effect of biochemical stimulants on biomass productivity and metabolite content of the microalga, Chlorella sorokiniana. Appl. Biochem. Biotechnol. 162, 2400-2414. doi: 10.1007/s12010-010-9012-9012

Ip, P., and Chen, F. (2005). Employment of reactive oxygen species to enhance astaxanthin formation in Chlorella zofingiensis in heterotrophic culture. Process Biochem. 40, 3491-3496. doi: 10.1016/j.procbio.2005. 02.014

Karasyova, T., Klose, E., Mensel, R., and Steinberg, C. (2007). Natural organic matter differently modulates growth of two closely related coccal green algal species. Environ. Sci. Pollut. Res. 14, 88-93. doi: 10.1065/espr2006. 06.317

Kilian, O., Benemann, C., Niyogi, K., and Vick, B. (2011). High-efficiency homologous recombination in the oil-producing alga Nannochloropsis sp. Proc. Natl. Acad. Sci. U.S.A. 108, 21265-21269. doi: 10.1073/pnas.1105861108 
Kim, S., Kim, H., Ko, D., Yamaoka, Y., Otsuru, M., Kawai-Yamada, M., et al. (2013). Rapid induction of lipid droplets in Chlamydomonas reinhardtii and Chlorella vulgaris by Brefeldin A. PLoS ONE 8:e81978. doi: 10.1371/journal.pone.0081978

Kobayashi, M., Hirai, N., Kurimura, Y., Ohigashi, H., and Tsuji, Y. (1997). Abscisic acid-dependent algal morphogenesis in the unicellular green alga Haematococcus pluvialis. Plant Growth Regul. 22, 79-85. doi: 10.1023/A:1005862809711

Kobayashi, M., Kakizono, T., and Nagai, S. (1993). Enhanced carotenoid biosynthesis by oxidative stress in acetate-induced cyst cells of a green unicellular alga, Haematococcus pluvialis. Appl. Environ. Microbiol. 59, 867-873.

Kobayashi, M., Todoroki, Y., Hirai, N., Kurimura, Y., Ohigashi, H., and Tsuji, Y. (1998). Biological activities of abscisic acid analogs in the morphological change of the green alga Haematococcus pluvialis. J. Ferment. Bioeng. 85, 529-531. doi: 10.1016/S0922-338X(98)80076-7

Kotzabasis, K., Hatziathanasiou, A., Bengoa-Ruigomez, M., Kentouri, M., and Divanach, P. (1999). Methanol as alternative carbon source for quicker efficient production of the microalgae Chlorella minutissima: role of the concentration and frequence of administration. J. Biotechnol. 70, 357-362. doi $10.1016 /$ S0079-6352(99)80128-3

Leu, S., and Boussiba, S. (2014). Advances in the production of high-value products by microalgae. Ind. Biotechnol. 10, 169-183. doi: 10.1089/ind.2013.0039

Li, Y., Sommerfeld, M., Chen, F., and Hu, Q. (2008). Consumption of oxygen by astaxanthin biosynthesis: a protective mechanism against oxidative stress in Haematococcus pluvialis (Chlorophyceae). J. Plant Physiol. 165, 1783-1797. doi: 10.1016/j.jplph.2007.12.007

Li, Y., Sommerfeld, M., Chen, F., and Hu, Q. (2010). Effect of photon flux densities on regulation of carotenogenesis and cell viability of Haematococcus pluvialis (Chlorophyceae). J. Appl. Phycol. 22, 253-263. doi: 10.1007/s10811-009-94539456

Linden, H. (1999). Carotenoid hydroxylase from Haematococcus pluvialis: cDNA sequence, regulation and functional complementation. Biochim. Biophys. Acto 1446, 203-212. doi: 10.1016/S0167-4781(99)00088-3

Liu, Z., Wang, G., and Zhou, B. (2008). Effect of iron on growth and lipid accumulation in Chlorella vulgaris. Bioresour. Technol. 99, 4717-4722. doi: 10.1016/j.biortech.2007.09.073

Lolke, S., Alistair, J., and Colin, R. (2005). "Alternative carbon sources for heterotrophic production of docosahexaenoic acid by the marine alga Crypthecodinium cohnii," in Single Cell Oils, eds C. Ratledge and Z. Cohen (Champaign, IL: AOCS Publishing), 131-149. doi: 10.1201/9781439822364.ch8

Lotan, T., and Hirschberg, J. (1995). Cloning and expression in Escherichia coli of the gene encoding $\beta$-C-4-oxygenase, that converts $\beta$-carotene to the ketocarotenoid canthaxanthin in Haematococcus pluvialis. FEBS Lett. 364, 125-128. doi: 10.1016/0014-5793(95)00368-J

Lu, Y., Jiang, P., Liu, S., Gan, Q., Cui, H., and Qin, S. (2010). Methyl jasmonate- or gibberellins A3-induced astaxanthin accumulation is associated with up-regulation of transcription of beta-carotene ketolase genes (bkts) in microalga Haematococcus pluvialis. Bioresour. Technol. 101, 6468-6474. doi 10.1016/j.biortech.2010.03.072

Lustigman, B., McCormick, J., Dale, G., and McLaughlin, J. (1987). Effect of increasing copper and salinity on glycerol production by Dunaliella salina. Bull. Environ. Contam. Toxicol. 38, 359-362. doi: 10.1007/bf01606687

Ma, R., and Chen, F. (2001a). Induction of astaxanthin formation by reactive oxygen species in mixotrophic culture of Chlorococcum sp. Biotechnol. Lett. 23, 519-523. doi: 10.1023/A:1010370401235

Ma, R., and Chen, F. (2001b). Enhanced production of free trans-astaxanthin by oxidative stress in the cultures of the green microalga Chlorococcum sp. Proces Biochem. 36, 1175-1179. doi: 10.1016/S0032-9592(01)00157-1

Mojaat, M., Pruvost, J., Foucault, A., and Legrand, J. (2008). Effect of organic carbon sources and $\mathrm{Fe} 2+$ ions on growth and $\beta$-carotene accumulation by Dunaliella salina. Biochem. Eng. J. 39, 177-184. doi: 10.1016/j.bej.2007.09.009

Navakoudis, E., Ioannidis, N., Dörnemann, D., and Kotzabasis, K. (2007). Changes in the LHCII-mediated energy utilization and dissipation adjust the methanol-induced biomass increase. Biochim. Biophys. Acta 1767, 948-955. doi: 10.1016/j.bbabio.2007.05.003

Norsker, N., Barbosa, M., Vermue, M., and Wijffels, R. (2011). Microalgal production-a close look at the economics. Biotechnol. Adv. 29, 24-27. doi: 10.1016/j.biotechadv.2010.08.005

Park, W., Yoo, G., Moon, M., Kim, C., Choi, Y., and Yang, J. (2013). Phytohormone supplementation significantly increases growth of Chlamydomonas reinhardti cultivated for biodiesel production. Appl. Biochem. Biotechnol. 171, 1128-1142. doi: 10.1007/s12010-013-0386-389

Patel, V., Maji, D., Singh, A., Suseela, M., Sundaram, S., and Kalra, A. (2013). A natural plant growth promoter, calliterpenone, enhances growth and biomass, carbohydrate, and lipid production in cyanobacterium Synechocystis PCC 6803. J. Appl. Phycol. 26, 279-286. doi: 10.1007/s10811-013-0103-107

Piotrowska, A., and Czerpak, R. (2009). Cellular response of light/darkgrown green alga Chlorella vulgaris Beijerinck (Chlorophyceae) to exogenous adenine- and phenylurea-type cytokinins. Acta physiol. plant. 31, 573-585. doi: 10.1007/s11738-008-0267-y

Piotrowska, A., Czerpak, R., Pietryczuk, A., Olesiewicz, A., and Wêdoowska, M. (2008). The effect of indomethacin on the growth and metabolism of green alga Chlorella vulgaris Beijerinck. Plant Growth Regul. 55, 125-136. doi: 10.1007/s10725-008-9267-9266

Raman, V., and Ravi, S. (2010). Effect of salicylic acid and methyl jasmonate on antioxidant systems of Haematococcus pluvialis. Acta Physiol. Plant. 33, 10431049. doi: 10.1007/s11738-010-0623-626

Rayle, D., and Cleland, R. (1992). The Acid Growth Theory of auxininduced cell elongation is alive and well. Plant Physiol. 99, 1271-1274. doi: 10.1104/pp.99.4.1271

Razon, L., and Tan, R. (2011). Net energy analysis of the production of biodiesel and biogas from the microalgae: Haematococcus pluvialis and Nannochloropsis. Appl. Energy 88, 3507-3514. doi: 10.1016/j.apenergy.2010.12.052

Ren, L., Huang, H., Xiao, A., Lian, M., Jin, L., and Ji, X. (2009). Enhanced docosahexaenoic acid production by reinforcing acetyl-CoA and NADPH supply in Schizochytrium sp. HX-308. Bioprocess Biosyst. Eng. 32, 837-843. doi: 10.1007/s00449-009-0310-314

Rioboo, C., González, Ó., Abalde, J., and Cid, Á. (2011). Flow cytometric analysis of the encystment process induced by paraquat exposure in Haematococcus pluvialis (Chlorophyceae). Eur. J. Phycol. 46, 89-97. doi: 10.1080/09670262.2011. 561775

Sarada, R., Tripathi, U., and Ravishankar, G. (2002). Influence of stress on astaxanthin production in Haematococcus pluvialis grown under different culture conditions. Process Biochem. 37, 623-627. doi: 10.1016/S0032-9592(01) 00246-1

Saygideger, S., and Deniz, F. (2008). Effect of 24-epibrassinolide on biomass, growth and free proline concentration in Spirulina platensis (Cyanophyta) under $\mathrm{NaCl}$ stress. Plant Growth Regul. 56, 219-223. doi: 10.1007/s10725-008-9310-9317

Saygideger, S., and Okkay, O. (2008). Effect of 2,4-dichlorophenoxyacetic acid on growth, protein and chlorophyll-a content of Chlorella vulgaris and Spirulina platensis cells. J. Environ. Biol. 29, 175-178.

Sheehan, J., Dunahay, T., Benemann, J., and Roessler, P. (1998). Look Back at the U.S. Department of Energy's Aquatic Species Program: Biodiesel from Algae. Golden: National Renewable Energy Laboratory.

Skjanes, K., Rebours, C., and Lindblad, P. (2013). Potential for green microalgae to produce hydrogen, pharmaceuticals and other high value products in a combined process. Crit. Rev. Biotechnol. 33, 172-215. doi: 10.3109/07388551.2012.681625

Soratana, K., and Landis, A. (2011). Evaluating industrial symbiosis and algae cultivation from a life cycle perspective. Bioresour. Technol. 102, 6892-6901. doi: 10.1016/j.biortech.2011.04.018

Steinbrenner, J., and Linden, H. (2001). Regulation of two carotenoid biosynthesis genes coding for phytoene synthase and carotenoid hydroxylase during stressInduced astaxanthinformation in the green alga Haematococcus pluvialis. Plant Physiol. 125, 810-817. doi: 10.1104/pp.125.2.810

Steinbrenner, J., and Linden, H. (2003). Light induction of carotenoid biosynthesis genes in the green alga Haematococcus pluvialis: regulation by photosynthetic redox control. Plant Mol. Biol. 52, 343-356. doi: 10.1023/a:1023948929665

Su, Y., Wang, J., Shi, M., Niu, X., Yu, X., Gao, L., etal. (2014). Metabolomic and network analysis of astaxanthin-producing Haematococcus pluvialis under various stress conditions. Bioresour. Technol. 170, 522-529. doi: 10.1016/j.biortech.2014.08.018.

Suen, Y., Hubbard, J., Holzer, G., and Tornabene, T. (1987). Total lipid production of the green alga Nannochloropsis sp. QII under different nitrogen regimes. J. Phycol. 23, 289-296. doi: 10.1111/j.1529-8817.1987.tb04137.x

Sun, Z., Cunningham, F., and Gantt, E. (1998). Differential expression of two isopentenyl pyrophosphate isomerases and enhanced carotenoid accumulation in a unicellular chlorophyte. Proc. Natl. Acad. Sci. U.S.A. 95, 11482-11488. doi: 10.1073/pnas.95.19.11482 
Sunohara, Y., and Matsumoto, H. (1997). Comparative physiological effects of quinclorac and auxins, and light involvement in quinclorac-induced chlorosis in corn leaves. Pestic. Biochem. Physiol. 58, 125-132. doi: 10.1006/pest.1997.2289

Theodoridou, A., Dörnemann, D., and Kotzabasis, K. (2002). Light-dependent induction of strongly increased microalgal growth by methanol. Biochim. Biophys. Acta 1573, 189-198. doi: 10.1016/S0304-4165(02)00438-5

Tripathi, U., Sarada, R., Rao, S., and Ravishankar, G. (1999). Production of astaxanthin in Haematococcus pluvialis cultured in various media. Bioresour. Technol. 68 , 197-199. doi: 10.1016/S0960-8524(98)00143-6

Vance, B. (1987). Phytohormone effects on cell division in Chlorella pyrenoidosa chick (TX-7-11-05) (chlorellaceae). J. Plant Growth Regul. 5, 169-173. doi: $10.1007 / \mathrm{bf} 02087185$

Vanthoor-Koopmans, M., Wijffels, R., Barbosa, M., and Eppink, M. (2013). Biorefinery of microalgae for food and fuel. Bioresour. Technol. 135, 142-149. doi: 10.1016/j.biortech.2012.10.135

Vidhyavathi, R., Venkatachalam, L., Sarada, R., and Ravishankar, G. (2008). Regulation of carotenoid biosynthetic genes expression and carotenoid accumulation in the green alga Haematococcus pluvialis under nutrient stress conditions. J. Exp. Bot. 59, 1409-1418. doi: 10.1093/jxb/ern048

Wang, J., Sommerfeld, M., and Hu, Q. (2009). Occurrence and environmental stress responses of two plastid terminal oxidases in Haematococcus pluvialis (Chlorophyceae). Planta 230, 191-203. doi: 10.1007/s00425-009-0932-934

Wijffels, R., Barbosa, M., and Eppink, M. (2010). Microalgae for the production of bulk chemicals and biofuels. Biofuels Bioprod. Biorefin. 4, 287-295. doi: 10.1002/bbb. 215

Wong, P. (2000). Effects of 2, 4-D, glyphosate and paraquat on growth, photosynthesis and chlorophyll-a synthesis of Scenedesmus quadricauda Berb 614 Chemosphere 41, 177-182. doi: 10.1016/S0045-6535(99)00408-7
Yen, H., Hu, I., Chen, C., Ho, S., Lee, D., and Chang, J. (2013). Microalgae-based biorefinery-from biofuels to natural products. Bioresour. Technol. 135, 166-174. doi: 10.1016/j.biortech.2012.10.099

Zhang, W., Li, F., and Nie, L. (2010). Integrating multiple 'omics' analysis for microbial biology: application and methodologies. Microbiology 156, 287-301. doi: 10.1099/mic.0.034793-34790

Zhu, X., Kong, H., Gao, Y., Wu, M., and Kong, F. (2012). Low concentrations of polycyclic aromatic hydrocarbons promote the growth of Microcystis aeruginosa. J. Hazard. Mater. 237-238, 371-375. doi: 10.1016/j.jhazmat.2012. 08.029

Conflict of Interest Statement: The authors declare that the research was conducted in the absence of any commercial or financial relationships that could be construed as a potential conflict of interest.

Received: 14 November 2014; accepted: 16 January 2015; published online: 17 February 2015.

Citation: $Y u$ X, Chen $L$ and Zhang $W$ (2015) Chemicals to enhance microalgal growth and accumulation of high-value bioproducts. Front. Microbiol. 6:56. doi: 10.3389/fmicb.2015.00056

This article was submitted to Food Microbiology, a section of the journal Frontiers in Microbiology.

Copyright (c) $2015 \mathrm{Yu}$, Chen and Zhang. This is an open-access article distributed under the terms of the Creative Commons Attribution License (CC BY). The use, distribution or reproduction in other forums is permitted, provided the original author(s) or licensor are credited and that the original publication in this journal is cited, in accordance with accepted academic practice. No use, distribution or reproduction is permitted which does not comply with these terms. 